\title{
Transcriptomic analysis reveals pronounced changes in gene expression due to sub- lethal pyrethroid exposure and ageing in insecticide resistance Anopheles coluzzii
}

\author{
V. A. Ingham ${ }^{1,2^{*}}$, F. Brown ${ }^{1,3}$ and H. Ranson ${ }^{1}$
}

\begin{abstract}
Background: Malaria control is heavily reliant on the use of insecticides that target and kill the adult female Anopheline vector. The intensive use of insecticides of the pyrethroid class has led to widespread resistance in mosquito populations. The intensity of pyrethroid resistance in some settings in Africa means mosquitoes can contact bednets treated with this insecticide class multiple times with minimal mortality effects. Furthermore, both ageing and diel cycle have been shown to have large impacts on the resistance phenotype. Together, these traits may affect other aspects of vector biology controlling the vectorial capacity or fitness of the mosquito.

Results: Here we show that sublethal exposure of a highly resistant Anopheles coluzzii population originally from Burkina Faso to the pyrethroid deltamethrin results in large and sustained changes to transcript expression. We identify five clear patterns in the data showing changes to transcripts relating to: DNA repair, respiration, translation, behaviour and oxioreductase processes. Further, we highlight differential regulation of transcripts from detoxification families previously linked with insecticide resistance, in addition to clear down-regulation of the oxidative phosphorylation pathway both indicative of changes in metabolism post-exposure. Finally, we show that both ageing and diel cycle have major effects on known insecticide resistance related transcripts.

Conclusion: Sub-lethal pyrethroid exposure, ageing and the diel cycle results in large-scale changes in the transcriptome of the major malaria vector Anopheles coluzzii. Our data strongly supports further phenotypic studies on how transcriptional changes such as reduced expression of the oxidative phosphorylation pathway or pyrethroid induced changes to redox state might impact key mosquito traits, such as vectorial capacity and life history traits.
\end{abstract}

Keywords: Insecticide resistance, Anopheles coluzzii, Pyrethroids, Transcriptome, Sub-lethal exposure

\footnotetext{
* Correspondence: Victoria.ingham@uni-heidelberg.de

'Department of Vector Biology, Liverpool School of Tropical Medicine, Liverpool L35QA, UK

${ }^{2}$ Present Address: Parasitology Unit, Centre for Infectious Diseases, Universitätsklinikum, Im Neuenheimer Feld 324, 69120 Heidelberg, Germany Full list of author information is available at the end of the article
}

(c) The Author(s). 2021 Open Access This article is licensed under a Creative Commons Attribution 4.0 International License, which permits use, sharing, adaptation, distribution and reproduction in any medium or format, as long as you give appropriate credit to the original author(s) and the source, provide a link to the Creative Commons licence, and indicate if changes were made. The images or other third party material in this article are included in the article's Creative Commons licence, unless indicated otherwise in a credit line to the material. If material is not included in the article's Creative Commons licence and your intended use is not permitted by statutory regulation or exceeds the permitted use, you will need to obtain permission directly from the copyright holder. To view a copy of this licence, visit http://creativecommons.org/licenses/by/4.0/ The Creative Commons Public Domain Dedication waiver (http://creativecommons.org/publicdomain/zero/1.0/) applies to the data made available in this article, unless otherwise stated in a credit line to the data. 


\section{Background}

Insecticide based vector control tools are the cornerstone of malaria control programmes and have proven to be the most efficient means for reducing malaria related morbidity and mortality since the turn of the century [1]. However, following dramatic reductions in malaria cases since 2000, progress has plateaued in the last 2 years [2]; a key driver of this is widespread insecticide resistance in Anopheline vectors [3-5]. Over 2 billion insecticide treated bed nets (ITNs) have been distributed in Africa, the WHO region accounting for the majority of the malaria burden worldwide; these nets are all treated with the pyrethroid class of insecticide. Resistance to pyrethroids is ubiquitous across sub-Saharan Africa. Indeed, of the reporting countries, almost $90 \%$ detailed pyrethroid resistance [2]. In some regions, the strength of this resistance allows mosquitoes to survive multiple bed net exposures with no observable impact on mosquito longevity [6]. Pyrethroid resistance reduces the personal protection provided by bed nets but also importantly erodes the community protection afforded to non-net users by insecticide induced mortality, which has been critical for their success [7-9]. To address this problem, net manufacturers have developed new classes of nets, several of which have already been pre-qualified by WHO and are now in use in Africa. Critically, these ITNs all still contain pyrethroid insecticides but their efficacy against pyrethroid resistant mosquitoes is enhanced by the presence of a second chemistry, either an insecticide, synergist or insect sterilising agent $[5,10]$. Hence, pyrethroids will remain an essential critical chemistry for malaria prevention for the foreseeable future and thus understanding the effects of pyrethroid exposure and pyrethroid resistance on Anopheles mosquitoes is of fundamental importance.

Pyrethroid resistance is multifactorial and is presently thought to be driven by four mechanisms; mutations to the target site of the pyrethroid insecticide, known as knockdown resistance (kdr) [11]; changes to the thickness of the mosquito cuticle that reduce penetrance of the insecticide [12]; sequestration by chemosensory proteins (CSPs) in the legs [13]; and finally, increased metabolic breakdown and clearance of the insecticide through over-expression of detoxification gene families [14-16]. Several members of the Anopheles cytochrome p450 family (P450s) have been shown to directly metabolise pyrethroids [16]; other detoxification gene families have also been implicated in resistance including glutathione-s-transferases (GSTs) [17], ABC transporters (ABCs) [18], carboxylesterases (COEs) [19] and UDPglucuronyl transferases (UGTs) [20]. All these mechanisms, with the exception of $k d r$, are caused by overexpression of specific members of these gene families within resistant mosquitoes and have been identified in multiple transcriptomic datasets comparing resistant and susceptible populations [21]. The large library of transcriptomic datasets available comparing resistant and susceptible mosquitoes represents a valuable resource for identifying resistance associated genes. However, these experiments were designed to remove potential confounding induction effects of pyrethroid exposure and in most cases mosquitoes were harvested for RNA extraction $48 \mathrm{~h}$ after exposure [21]. The process of correcting for induction effects loses data about how insecticide exposure could potentially affect mosquito biology and behaviour within this window. These facets of the mosquito response are important to investigate both to understand the mechanisms underpinning any post exposure behavioural changes such as willingness to blood feed [22] and to predict potential impacts of insecticide exposure on the development of the malaria parasite in the mosquito.

Previous studies have looked at the induction effects of insecticides on specific genes of interest and shown that both constitutive overexpression and induction are important in response to insecticide exposure. These studies include pyrethroid induction of cytochrome p450s in Cx. quinquefasciatus $[23,24]$ and D. melanogaster [25], ABC transporters in An. stephensi [26], CSPs in An. gambiae [13], COEs in Musca domestica [19], UGTs in Spodeoptera exigua [27] and GSTs in Bactrocera dorsalis [28]. Many of these insecticide-induced changes in transcript expression are linked with oxidative stress and the $c n c-N r f 1$ pathway, which has been shown to be constitutively up-regulated in insecticide resistance An. gambiae and D. melanogaster [29-31]. As far as we are aware, no studies have looked at overall change in the whole transcriptome over an extended time course; this is important to understand the molecular response to sub-lethal insecticide exposure.

In this study we exposed 3-day old An. coluzzii females from a highly resistant colony established from Burkina Faso [32] to the pyrethroid insecticide deltamethrin and investigated changes in the transcriptome over a $72 \mathrm{~h}$ time course. We identified five stages to the pyrethroid response, including a sustained change in genes associated with respiratory function transcription, behaviour, DNA damage and translation. The experimental design also captures the effects of both ageing and diel cycle and reveals multiple genes previously associated with insecticide resistance are differentially expressed following pyrethroid exposure, ageing and throughout the diurnal cycle.

\section{Results}

The experiments were designed to test three separate hypotheses: (i) Pyrethroid exposure induces changes to transcript expression over time; (ii) Ageing increases 
susceptibility to insecticides due to changes in expression of insecticide related transcripts and (iii) Diel cycle controls the expression of insecticide resistance transcripts. All experiments used the pyrethroid resistant VK7 strain of Anopheles coluzzii, originally colonised from Burkina Faso [32].

\section{Pyrethroid exposure induces changes to transcript expression over time}

To identify changes in transcript expression associated with pyrethroid exposure, 3-day old females were exposed to $0.05 \%$ deltamethrin papers for $1 \mathrm{~h}$ using a standard WHO tube assay. Mosquitoes were then harvested for RNA extraction at 10 time points post exposure: $0 \mathrm{~min}, 30 \mathrm{~min}, 1-\mathrm{h}, 2-\mathrm{h}, 4-\mathrm{h}, 8-\mathrm{h}, 12-\mathrm{h}, 24-\mathrm{h}, 48-\mathrm{h}$ and 72-h (Fig. 1). A total of 9041 transcripts (9547 probes) showed differential expression compared to an unexposed control (taken before exposure) in at least one of these time points. Two separate analyses were then used. Firstly, significance-independent soft clustering of these transcripts by temporal changes in expression was performed using Mfuzz with 20 clusters

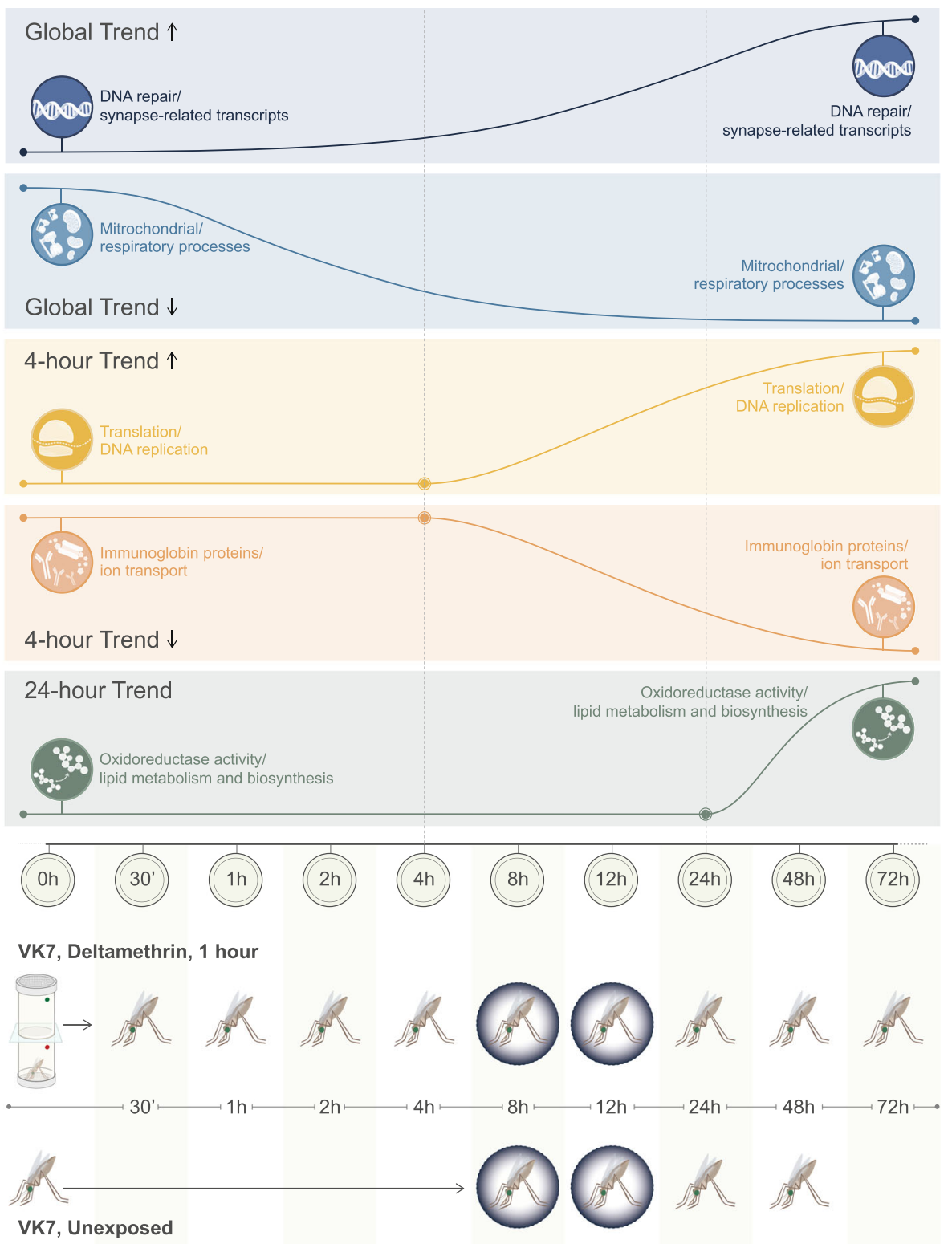

Fig. 1 Time series trends. Five rows demonstrating the temporal transcript pattern change and the associated enrichments for each trend. Experimental design is shown on the bottom two rows, with the time points (i) post $0.05 \%$ deltamethrin WHO tube exposure and (ii) matched unexposed controls. Dark rings represent darkness in the 12:12 photoperiod 
(Additional file 1; Additional file 2). Exploration of these clusters using enrichment analyses identified five key trends within the dataset (Fig. 1; Additional file 2). Secondly, transcripts showing significant differential expression following the same directionality over multiple time points were extracted; these transcripts are likely to represent the most important in the response to insecticide exposure.

Six of the 20 clusters showed sustained changes in the expression profiles after sublethal exposure to pyrethroids. These clusters include cluster 8,11 and 17 which represents transcripts with a trend for sustained up-regulation, whilst clusters 18,9 and 20 show the converse trend (Additional file 1). Cluster 8 is enriched for DNA repair related transcripts $(p=4.8 \mathrm{e}-2)$ and clusters 11 and 17 are enriched in neuronal related transcripts such as synapse assembly (8.4e-3), neurexin family protein binding (7.2e-3) and olfactory receptor activity (3.3e-2). The converse, cluster 9 (which shows the clearest pattern of sustained down regulation) is enriched in mitochondrial electron transport chain $(p=1.7 \mathrm{e}-6)$ and oxidative phosphorylation $(p=2 \mathrm{e}-9)$ consistent with oxidative damage and the associated reactive oxygen species burst shown to be caused by pyrethroid exposure in mammalian systems [33]. Clusters 18 and 20 are similarly enriched in glycolytic processes including carbon metabolism $(p=4 \mathrm{e}-8$; $8.4 \mathrm{e}-6)$, glycolysis $(p=7.2 \mathrm{e}-5)$, oxidative phosphorylation $(p=1.9 \mathrm{e}-9)$ and citrate (TCA) cycle $(p=5.2 \mathrm{e}-8)$ and indicative of an overall reduction in respiration post-insecticide exposure (Fig. 1; Additional file 2).

Transcripts demonstrating consistent and significant up-regulation across all time points are listed in Fig. 2 and include the ortholog of the $p 53$ transcription factor (AGAP002352-RB), which responds to genotoxic stress [34], AGAP001116-RA a D-amino acid oxidase linked with hydrogen peroxide production and detoxification, the UDP-transferase UGT308G1 (AGAP007990-RA), and the homolog of galla-1 (AGAP007363-RA). Significant
AGAP001116-RA
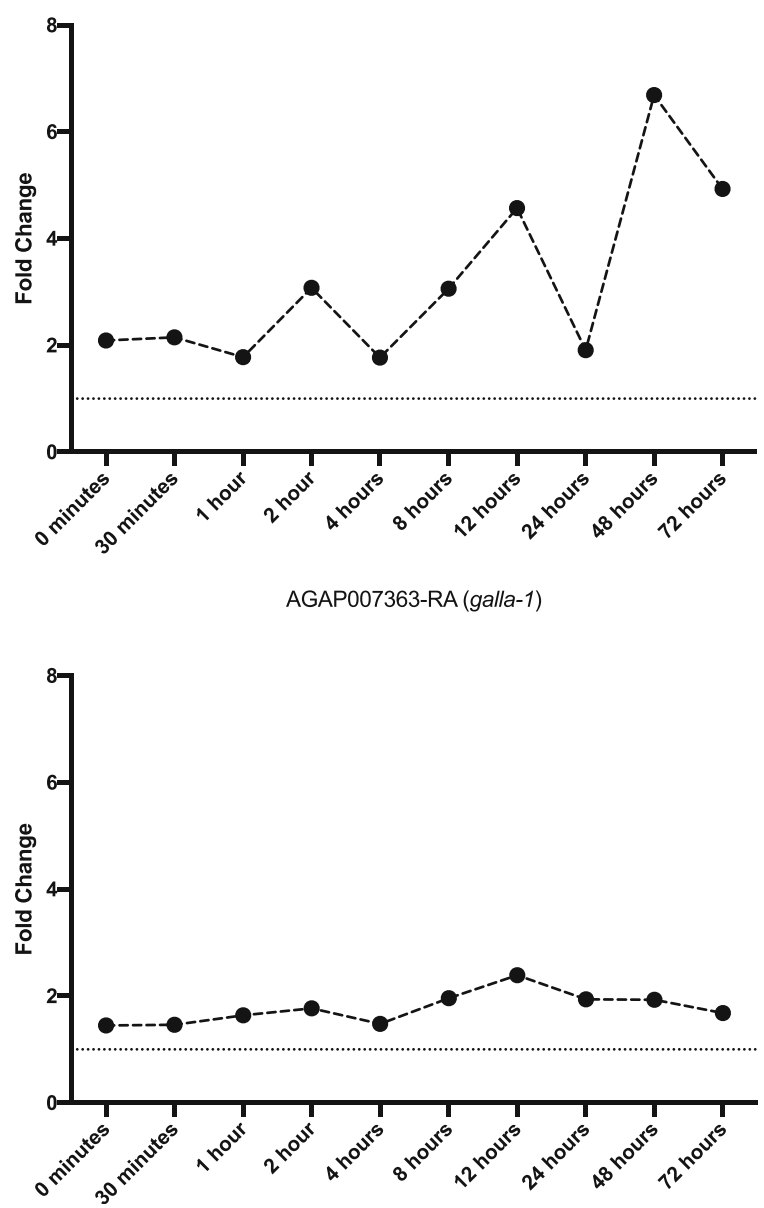

AGAP002352-RB ( $p 53)$

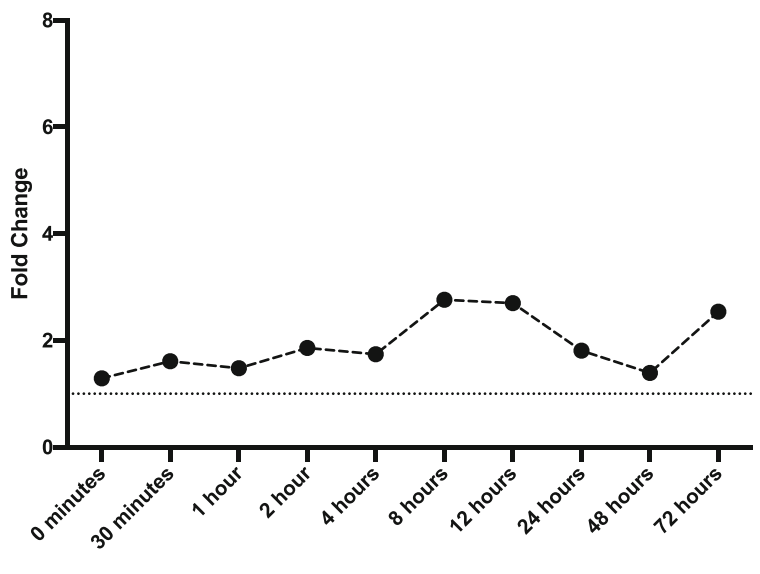

AGAP007990-RA (UGT308G1)

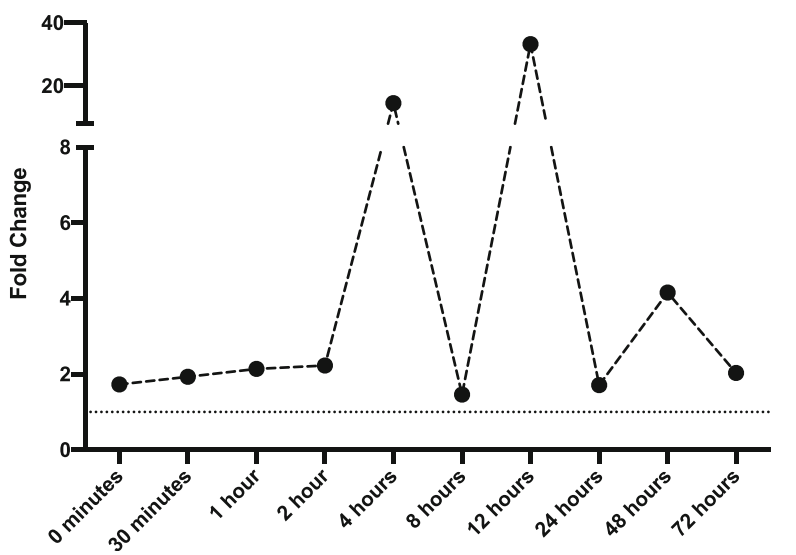

Fig. 2 Transcripts significant across all time points. Expression levels as fold changes (y) across all time points (x) for each transcript significantly differentially expressed across all time points (adjusted $p<0.05$ ). Titles of the graphs include both transcript IDs and gene names 
sustained down regulation across all time points was seen in 15 transcripts, including GSTZ1 (Additional file 3).

Five clusters grouped transcripts with clear changes in expression from 4-h post-exposure (Additional file 1). Cluster 14 shows sharp down regulation $4 \mathrm{~h}$ postexposure and is highly enriched in immunoglobin-like proteins $(p=2.2 \mathrm{e}-9)$, calcium ion binding $(p=6 \mathrm{e}-4)$ and neuroactive ligand-receptor interaction $(p=3.3 \mathrm{e}-4)$; a number of these transcripts belong to a class of proteins called defective proboscis extension response. In Drosophila these proteins are neuronal and have been shown to be involved in responses to chemical stimuli and stress [35]. Similarly, 2 protein orthologs of Drosophila sidestep are represented in this cluster along with the interactor beat both of which have been linked to oxidative stress response induced locomoter defects [36]. Conversely, clusters 15 and 12 demonstrate strong induction of transcripts from $4 \mathrm{~h}$ post-exposure and are enriched in translation $(p=2.5 \mathrm{e}-2)$ and structural components of the ribosome $(p=1.5 \mathrm{e}-2)$ cluster 4 similarly shows induction from $4 \mathrm{~h}$ post-exposure and is enriched in translation initiation factor activity $(p=2.3 \mathrm{e}-2)$ indicating the onset of protein production related to insecticide exposure (Fig. 1; Additional file 2).

Of those transcripts showing consistent significant expression directionality, 18 transcripts show a sustained, up-regulation from $1 \mathrm{~h}$ or $2 \mathrm{~h}$ post-exposure until $72 \mathrm{~h}$ post exposure (Additional file 4), including the transcription factor $\operatorname{Dr}$ (AGAP003669-RA), which plays a role in locomotor activity and neuronal patterning in Drosophila and IMD a key immune-response regulator (AGAP004959-RB). A larger number of transcripts (256; 266 probes), show a delayed but sustained induction response beginning at 4- or $8-\mathrm{h}$ post exposure (Additional file 5). Of the 20 transcripts showing delayed, sustained down regulation from 1 or $2 \mathrm{~h}$ onwards four are cuticular proteins (CPLCG3, 4 and 15 and CPR109); this transcript list also includes the D7r2 salivary protein (Additional file 4). A further 131 transcripts (140 probes) show sustained down-regulation either 4 or 8 $h$ post exposure (Additional file 5). Similarly, these transcripts contain a number of cuticular related transcripts including CPR10, CPLCA1, CPLCX3, CPR59, CPCFC1 and CPR132 and alternative probes for CPCLG4.

One cluster shows clear up-regulation of transcripts from 24-h post-exposure (no clusters show a pattern of down-regulation after this time point although 43 transcripts show significant down-regulation from 24-h onwards and 20 show significant up-regulation (Additional file 6)). Cluster 7 is enriched in oxidoreductase activity $(p=6.2 \mathrm{e}-3)$ and cytochrome 4450 domains $(p=$ 4.7e-3). Cluster 7 also shows a similar expression pattern showing changes in transcripts related to fatty acid degradation ( $p=1.3 \mathrm{e}-2)$ (Fig. 1); indicating that exposure to insecticide may lead to long term up-regulation of detoxification transcripts and differential expression of fatty acids (Fig. 1; Additional file 2).

Three other clusters show strong enrichments but do not show a strong sustained temporal expression change. Clusters 19, 6 and 5 show a peak of expression at 48-, 12-h and 8 and 12-h respectively (Additional file 1; Additional file 2). Clusters 5 and 6 are likely to represent strong circadian changes and are enriched in response to insecticide $(p=2.4 \mathrm{e}-2)$, cytochrome $\mathrm{p} 450 \mathrm{~s} \quad(p=1.2 \mathrm{e}-7)$ and digestion $(p=2.5 \mathrm{e}-2)$, likely reflecting the diel nature of expression of metabolic enzymes, described below. Cluster 19 is enriched in glutathione metabolic process, oxioreductase activity and cytochrome $\mathrm{p} 450 \mathrm{~s}(p=2.5 \mathrm{e}-3$; $9.4 \mathrm{e}-3 ; 1.2 \mathrm{e}-2)$ indicating changes relating to insecticide response peak strongly at 48-h.

\section{Induction of gene families associated with pyrethroid resistance}

Of 113 cytochrome p450s in the Anopheles genome, 82 are differentially expressed in at least one timepoint post pyrethroid exposure (Additional file 7). Of the 8 cytochrome p450s that bind to pyrethroid insecticides and have been widely implicated in pyrethroid resistance [16, 37] (Fig. 3), two, (CYP6M2 and CYP6Z2), are strongly induced after deltamethrin exposure. Closely related P450s not previously associated with pyrethroid metabolism are also strongly induced (CYP6M1, CYP6M3, CYP6Z3) (Additional file 7). Several other p450s are induced over multiple hours or days, including CYP4G16 and CYP4G17, both linked with cuticular thickening [12], CYP4D17, CYP6AH1, CYP6Z1 and CYP4C27. Notable genes from other detoxification gene families that are induced post exposure include, GSTD1, ABCG5 (previously shown to enriched in the abdomen and upregulated across multiple resistant population [18]), $A B C C 14$ (the homolog of Drosophila multidrug resistance protein 1 and up-regulated in multiple resistant populations [18]), COE13O and UGT308G1. CSPs have recently been linked with pyrethroid resistance in West Africa [13]; $S A P 3$, is highly induced from $8 \mathrm{~h}$ (Additional file 7) whereas $S A P 2$ is significantly over expressed at immediately post-exposure and $8 \mathrm{~h}$.

Trends of down-regulation are also seen within the detoxification families, including GSTZ1 (-RA only) which was down-regulated across all time points. Other transcripts showing down regulation across multiple time points include two transcripts labelled as CYP9M1 (AGAP009374-RA and AGAP009363-RA), CYP4H18, GSTD12, GSTE1, GSTE2, GSTMS3, GSTS1, ABCC7, COEAE5G and UGT301E2. Interestingly, GSTE2 has been strongly linked with DDT and pyrethroid resistance [17]; however, in this strain it is strongly down regulated from 4 to $48 \mathrm{~h}$ post-exposure. 


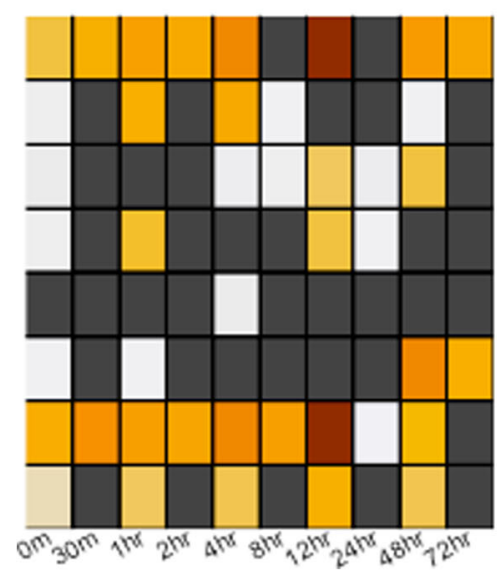

AGAP008212-RA (CYP6M2)

AGAP002865-RA (CYP6P3)

AGAP002869-RA (CYP6P2)

AGAP002867-RA (CYP6P4)

AGAP002866-RA (CYP6P5)

AGAP012296-RA (CYP9J5)

AGAP008218-RA (CYP6Z2)

AGAP000818-RA (CYP9K1)

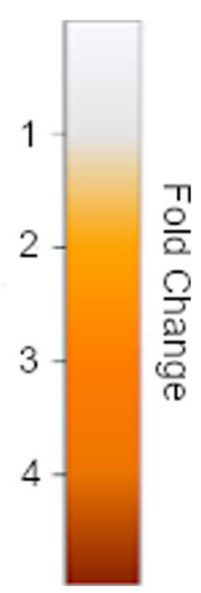

Fig. 3 Cytochrome p450 pyrethroid metabolisers. Transcript expression level for 8 cytochrome p450s that have previously been shown to bind insecticide directly $[16,37,38]$. Dark grey boxes represent non-significant transcripts

\section{Changes to respiratory-related transcripts}

As shown using soft clustering, transcripts demonstrating sustained downregulation post-pyrethroid exposure are enriched in transcripts involved in both the mitochondrial oxidative phosphorylation chain and in the TCA cycle, indicating a wider change to respiration caused by pyrethroid exposure. The changes in gene expression are shown in Additional file 8 and Fig. 3 provides a pictorial representation of the oxidative phosphorylation chain from which it can be seen that pyrethroid exposure suppresses gene expression for each of the five members of the respiratory complex (Fig. 4).

\section{Ageing increases susceptibility to insecticides due to changes in expression of insecticide related transcripts} A total of 931 transcripts (1033 probes; representing $6.93 \%$ of the array) were significantly differentially regulated between the 0 - $\mathrm{h}$ ( 3 day old) and 48-h (5 day old) timepoints in unexposed mosquitoes, indicating the extensive changes in gene expression as female mosquitoes age (Additional file 9). Of these, 403 transcripts (449 probes) were up-regulated and 528 (584 probes) down-regulated. Both up and down regulated genes showed significant enrichment for GO terms related to detoxification. Genes involved in heme-binding ( $p=$ $0.0085)$, glutathione transferase $(p=0.0011)$ and glutathione peroxidase activity $(p=0.049)$ were up-regulated whereas downregulated genes were enriched in insecticide catabolic process $(p=0.000494)$, mono-oxygenase activity $(p=3.8 \mathrm{e}-6)$, iron-binding $(p=2.2 \mathrm{e}-7)$, oxidoreductase activity $(p=1.5 \mathrm{e}-5)$, heme-binding $(p=1.5 \mathrm{e}-5)$ and organic anion transporter $(p=2.6 \mathrm{e}-4)$. A number of detoxification genes (274 transcripts, representing $<2 \%$ of the genome) (Table 1), chemosensory proteins [13] and a cuticular protein [40] previously linked with insecticide resistance are expressed a lower levels in 5 day females than 3 day olds, perhaps providing an explanation for previous observations that resistance to pyrethroid insecticide falls with mosquito age [41, 42]. However, some resistance-related transcripts, including methoprene tolerant [21], several members of the GSTD and GSTE families and CSP6 [13] are up-regulated in older mosquitoes.

\section{Diel cycle controls the expression of insecticide resistance} transcripts

Anopheles mosquitoes are night biting mosquitoes and, as adults, are most likely to encounter insecticide when searching for a blood meal in the evening. To assess the diel nature of insecticide related transcripts in a multiinsecticide resistant population, age matched females were compared in two steps (i) comparing transcript expression in mosquitoes sacrificed at $7 \mathrm{pm}$ or $11 \mathrm{pm}(8$ $\mathrm{h}$ and $12 \mathrm{~h}$ ) and (ii) $11 \mathrm{pm}$ or $11 \mathrm{am}$ (12 h and $24 \mathrm{~h}$ ) (Fig. 1). No transcripts showed a significantly differential expression pattern in group (i); however, in group (ii) 506 (587 probes) transcripts showed differential expression (Additional file 10; Table 2). Of these 230 (273 probes) were up-regulated and 276 (314 probes) were downregulated in the morning compared to the evening. Transcripts overexpressed in the morning were enriched in oxidoreductase and monooxygenase activity $(p=2.6 \mathrm{e}-$ 5 ; $3.5 \mathrm{e}-5)$; heme binding $(p=3.9 \mathrm{e}-5)$; iron ion biding $(p=2.04 \mathrm{e}-4)$ and both glutathione peroxidase and transferase activity $(p=3.54 \mathrm{e}-4 ; p=8.1 \mathrm{e}-4)$. Transcripts overexpressed in the evening were similarly enriched in oxidoreductase and monoxygenase activity ( $p=6 \mathrm{e}-4$; $9.3 \mathrm{e}-4)$, heme binding $(p=6.5 \mathrm{e}-4)$, iron ion binding ( $p=$ $5.4 \mathrm{e}-4)$ and insecticide catabolic process $(p=0.0014)$. Within these transcripts are direct insecticide interactors 


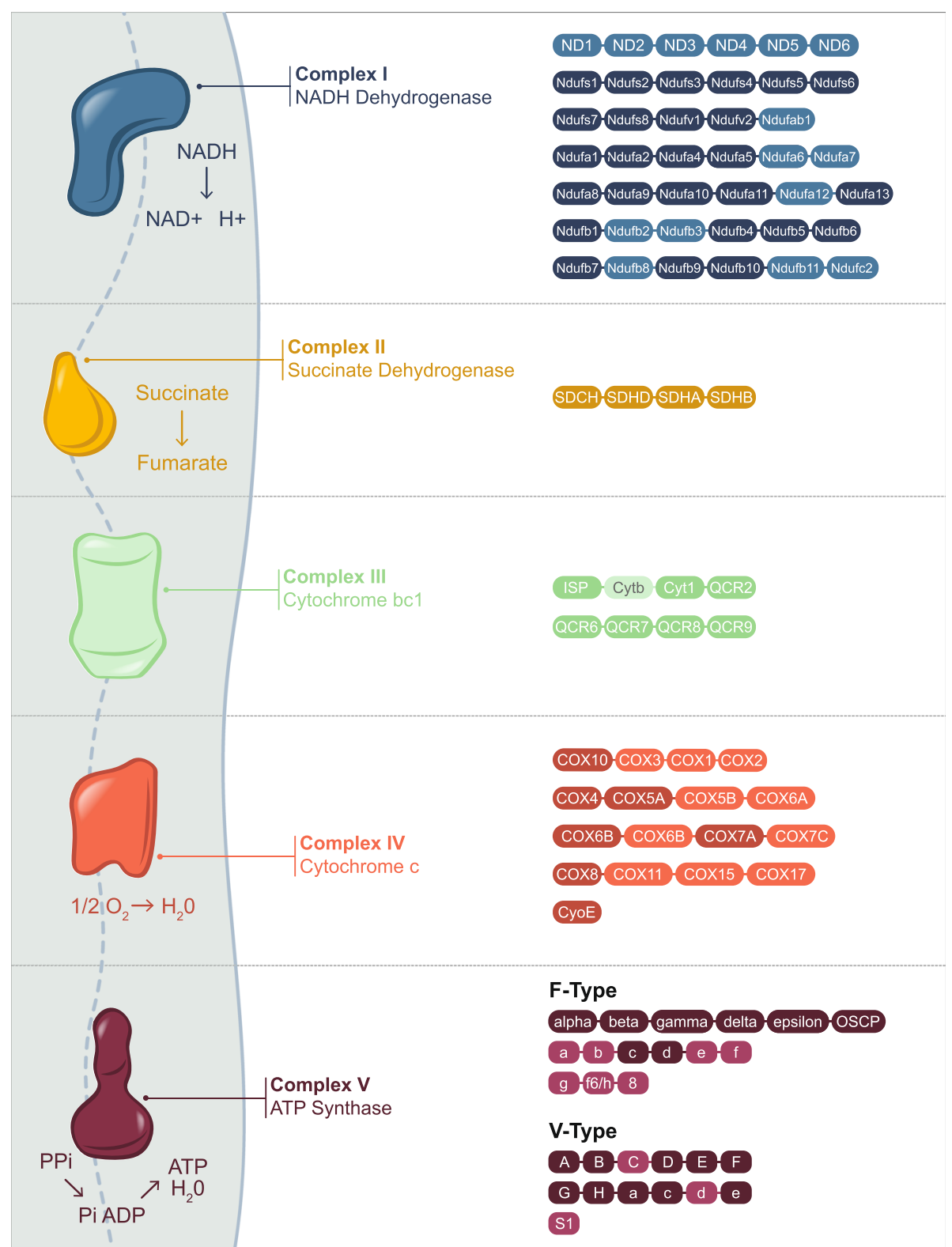

Fig. 4 Transcripts in the oxidative phosphorylation pathway down regulated by pyrethroid exposure. Modified KEGG pathway showing all transcripts in the oxidative phosphorylation in An. gambiae (KEGG organism: aga). Darkened boxes represent transcripts that are significantly down regulated in at least one time point in the time course data. ND1-6 are not represented on the microarray as they are mitochondrial genes

including SAP2 [13], CYP6M2 [15, 16], CYP6P3 [14, 16], CYP6P4 [16], CYP6Z1 [39] and CYP9K1 [38] (Table), all of which are expressed at higher levels at $11 \mathrm{pm}$ than 11 am as seen in previously published data [43]. As detoxification-related transcripts are enriched both in the morning and in the evening, this could indicate a two-phase process in metabolic clearance of pyrethroid insecticides with a subset of cytochrome p450s catalysing the initial oxidation reaction being highly enriched at night $(p=3.05 \mathrm{e}-7 ; 0.013)$, and a separate subset of cytochrome p450s, plus COEs and GSTs, responsible for secondary pyrethroid metabolism enriched in the morning $(p=4.37 \mathrm{e}-10 ; 5.2 \mathrm{e}-3 ; 2.56 \mathrm{e}-8)$, in agreement with a previous publication showing GST activity peaks at 22.1 Zeitgeber time [43]. Further, Anopheline antiplatelet protein, four salivary gland related proteins and several trypsin transcripts are enriched at night-time when the mosquito would be seeking a bloodmeal. Cycle and Clock are upregulated whilst Period, Cryptochrome 2, Timeless and PDP1 are downregulated in this dataset, confirming the rhythmic nature of these changes following the pattern previously reported in An. gambiae [44]. Indeed, these 
Table 1 Cytochrome p450s down regulated in 5 day vs 3 day old females. Transcript ID, Gene Name, Adjusted $p$-value and absolute Fold Change of cytochrome p450s previously implicated in insecticide resistance [14-16, 21, 39] in 5-day old adult female mosquitoes compared to 3-day old. Asterisk's indicates pyrethroid metabolising enzymes [14-16]

\begin{tabular}{llll}
\hline Transcript ID & Gene Name & Adjusted p-value & Fold Change \\
\hline AGAP000088-RA & CYP4H19 & 0.012 & 0.294 \\
AGAP000818-RA & CYP9K1* & 0.013 & 0.447 \\
AGAP000877-RA & CYP4G17 & 0.042 & 0.556 \\
AGAP001076-RA & CYP4G16 & 0.036 & 0.634 \\
AGAP001076-RB & CYP4G16 & 0.050 & 0.646 \\
AGAP001076-RC & CYP4G16 & 0.045 & 0.721 \\
AGAP002862-RA & CYP6AA1 & 0.049 & 0.590 \\
AGAP002865-RA & CYP6P3* & 0.006 & 0.379 \\
AGAP002894-RA & CYP6Z4 & 0.041 & 0.747 \\
AGAP008212-RA & CYP6M2* & 0.004 & 0.382 \\
AGAP008213-RA & CYP6M3 & 0.009 & 0.451 \\
AGAP008214-RA & CYP6M4 & 0.043 & 0.739 \\
AGAP008217-RA & CYP6Z3 & 0.042 & 0.524 \\
AGAP008218-RA & CYP6Z2* & 0.029 & 0.373 \\
AGAP008219-RA & CYP6Z1 & 0.008 & 0.336 \\
AGAP013490-RA & CYP4H24 & 0.013 & 0.275 \\
\hline
\end{tabular}

data show a large overlap with genes previously found to be rhythmic, with > 55\% concordance [44].

\section{Discussion}

Insecticide resistance has been defined by WHO as the number one obstacle to malaria elimination. The majority of studies investigating the molecular basis of resistance focus on the constitutive overexpression of transcripts in resistant mosquito populations, compared to susceptible controls; however, induction of gene expression after a sub-lethal dose of insecticide is likely to be equally important for long term fitness effects and parasite transmission. In this study, we explore these

Table 2 Direct pyrethroid interactors upregulated at night. Transcript ID, Gene Name, Adjusted p-value and absolute Fold Change of transcripts that directly interact with insecticides showing enrichment at $11 \mathrm{pm}$ compared to $11 \mathrm{am}$ (downregulation at $11 \mathrm{am}$ compared to $11 \mathrm{pm}$ as shown above)

\begin{tabular}{llll}
\hline Transcript ID & Gene Name & Adjusted p-value & Fold Change \\
\hline AGAP000818-RA & CYP9K1 & 0.007 & 0.543 \\
AGAP002865-RA & CYP6P3 & 0.004 & 0.400 \\
AGAP002867-RA & CYP6P4 & 0.026 & 0.531 \\
AGAP008052-RA & SAP2 & 0.018 & 0.473 \\
AGAP008212-RA & CYP6M2 & 0.012 & 0.448 \\
AGAP008219-RA & CYP6Z1 & 0.024 & 0.614 \\
\hline
\end{tabular}

factors using whole transcriptome microarrays with a pyrethroid-resistant An. coluzzii population, VK7. This strain was selected as the high levels of pyrethroid resistance are conferred by multiple mechanisms including target site mutations and high levels of overexpression of the cytochrome p450 pyrethroid metabolisers CYP6M2 and CYP6P3 [32] with further, less well characterised resistance mechanisms potentially associated with increased expression of $\alpha$-crystallins and an F-Type ATPase [32].

In this study we identify five phases to the response to pyrethroid exposure. There is an immediate and sustained reduction in genes involved in mitochondrial respiration and a sustained increase in expression of DNA-damage response related and behaviour related transcripts. A reduced respiratory rate after exposure to pyrethroid insecticides has been widely reported in mammalian and fish systems through inhibition of the oxidative phosphorylation chain [45-47]; however, as far as we are aware, no studies have examined this in insects. It is possible a reduction in respiration postpyrethroid exposure represents a compensatory mechanism to reduce mitochondrially produced reactive oxygen species due to exogenous ROS production from sublethal pyrethroid exposure [48]. Similarly, the oxidative stress caused by pyrethroid exposure are likely to cause genotoxicity $[48,49]$, hence explaining the up-regulation of DNA-repair related transcripts. We further show that 4-h post exposure there is a large change in transcription with up-regulation of translation and down regulation of ion transport and immunoglobin-like proteins. The up-regulation of transcripts related to translation could be due to the sustained changes seen in transcriptional activity for up to $72 \mathrm{~h}$ resulting in higher levels of protein production. Further, the down-regulation of neuronal-related transcripts could account for some behavioural changes seen in Anopheles mosquitoes on contact with ITNs [50, 51]. Perhaps most surprisingly, enrichment in monooxygenase activity, cytochrome P450 domains and glutathione activity, commonly associated with insecticide resistance are only induced after 24-h post exposure. The induction of these transcripts from a day post-exposure across all subsequent time points suggests that sub-lethal exposure could lead to higher levels of resistance upon second exposure the following night through overactivity of detoxification related transcripts. Indeed, this has been demonstrated after mosquitoes take a bloodmeal [52], which induces a large oxidative stress response, similar to those seen in mammalian systems after pyrethroid exposure.

Five transcripts show differential expression from immediately after pyrethroid exposure to the maximal timepoint, $72 \mathrm{~h}$. These transcripts are likely to be some of the most important for insecticide response and contain $p 53$, a DNA-damage related transcription factor 
[34], UGT308G1, the homolog of galla-1 and a transcript linked with reactive oxygen species response, a D-aminoacid oxidase [53]. $p 53$ has been shown to have multiple roles in cellular response to genotoxic stress in Drosophila [34]. Few studies have explored the function of these genes in Anopheles mosquitoes. One study on mosquito p53 orthologs describes a direct role in response to oxidative stress upon arboviral infection [54], perhaps indicating that this gene may respond to similar stress post-pyrethroid exposure. Similarly, the UGT family have previously been linked to insecticide resistance [20], the high level of induction of this transcript across all timepoints suggests that the role of this family in pyrethroid detoxification merits further study. Indeed, the UGT308 family has been shown to be an essential family for the biotransformation of pyrethroid insecticides in An. sinensis, an Asian malaria vector [55]. A SNP in galla-1 was found to be significantly associated with Drosophila response to oxidative stress [36] and was also found to play a role in protective response to reactive oxygen species in fragile genetic sites through alterations to aerobic metabolism [56]. The Drosophila homolog of the D-amino-acid oxidase described here is localised to the peroxisome, these membrane bound organelles play a key role in both the production and detoxification of cellular reactive oxygen species [53]. Taken together, there is strong indication that these transcripts play a key role in response to oxidative stress caused by exposure to pyrethroid insecticides, either through maintaining cellular homeostasis or protection of genetic material. The down-regulated transcripts include a number of cuticular proteins and the $\mathrm{ABC}$ transporter $\mathrm{ABCH} 2$, a half transporter whose role in insects is poorly characterised [18]. Interestingly, the salivary protein $D 7 r 2$ has previously been linked to bendiocarb resistance through ubiquitous overexpression [57]; however, these data indicate that this transcript may not be important in pyrethroid resistance in this population, supported by recently published data from An. gambiae in Cameroon [58].

The induction of detoxification candidates previously shown to be involved with insecticide resistance is an important consideration, as many of these transcripts are already expressed at constitutively higher levels within pyrethroid resistant mosquito populations. For example, CYP6M2 is 8 fold constitutively overexpressed in VK7 compared to a susceptible control [32], and here we show a further 5.2 fold overexpressed maximally post-exposure. Similarly, CYP6Z2 is $3.5 \mathrm{x}$ constitutively overexpressed [59] and a further $7.2 x$ maximally induced, clearly demonstrating the importance of induction of these pyrethroid metabolisers.

Pyrethroid resistance has previously been shown to fall with age [41, 42], in the absence of a blood meal [52]. To explore the transcriptional basis of this reduction, we compared 3- and 5-day old mosquitoes and found substantial changes in transcript expression. Of the transcripts down regulated were a number of genes previously linked to insecticide resistance such as, a cuticular protein CPLCG5 [40], CYP6P3 [14], CYP4G16 [12] and the chemosensory protein SAP2 [13]. Given the relatively large reductions in transcript expression over a short time period, it is likely that the reduction in expression of these key transcripts play a large role in the relative loss of resistance; however, further time points, with accompanying bioassays, would be needed to investigate further. Here, 931 transcripts were significantly differentially express over a two-day period, representing $7.0 \%$ of the transcriptome. Studies in other mosquito species saw similar trends; one in Aedes mosquitoes which shows $10.8 \%$ of transcripts change with a fold change cut off of 2 , between $24 \mathrm{~h}$ and $72 \mathrm{~h}$ female carcasses and a second in Anopheles gambiae which shows 20.3\% of transcripts following this trend over a 2 day period [60, 61].

Previous data has demonstrated a high number of transcripts are controlled by the diel cycle and circadian clock in susceptible An. gambiae mosquitoes, with an enrichment of those involved in detoxification [44]; here we show that CYP6Z1, CYP6P3 and CYP6M2 showed peak expression during the evening [44] and $S A P 2$, a key pyrethroid resistance determinant in West Africa, is also upregulated at night. There is also a clear enrichment for oxidoreductase activity early morning, which may indicate a potential two-step process in metabolic clearance of insecticides, with the first being direct metabolism through cytochrome p450s and binding and the second stage related to clearance of the metabolites through up-regulation of GSTs. The primary p 450 response followed by a later GST response, potentially clearly insecticide metabolites, is in line with a previous study examining the changes in activity of these genes throughout the 24-h cycle [43]. To further investigate the rhythmic changes in transcript expression in this resistant population, more time points would be necessary; instead, these data present a snapshot of the changes in expression at the likely maximal and minimal point of the circadian cycle and are largely in agreement with previous studies [43, 44].

Although this study provides a detailed picture of sublethal pyrethroid exposure in a resistant An. coluzzii population from Burkina Faso, it is clear that even within country there are differing resistance mechanisms between sites [32]. Further, the 1-h WHO exposure used here is not representative of pyrethroid dose received under natural settings, where mosquitoes spend shorter time in contact with higher concentrations on insecticide treated surfaces [50].

\section{Conclusion}

This study provides insight into the large changes in transcript expression at various time-points post-pyrethroid exposure. The sustained transcriptional changes seen here are likely to have important phenotypic effects; for 
example, the reduced respiratory rate, and increased investment in DNA repair and protein production are likely to have energetic costs which may reduce the fitness of mosquitoes' post pyrethroid exposure. Although no shortening of longevity is seen in this population after insecticide exposure in a laboratory environment [6], other fitness traits such as fertility and fecundity merit further studies. Further, if pyrethroid exposure impacts the redox state of the mosquito, as indicated by the widespread changes to oxidoreductase-related transcripts and respiratory rate, this may impact the mosquito's ability to transmit pathogens. Disruption of parasite development due to changes in redox state has been shown experimentally through reducing catalase activity which in turn reduces oocyst density in the midgut [62], whilst the initial immune response to parasite invasion is a large reactive oxygen species burst $[62,63]$. Hence, future studies should investigate how pyrethroid exposure affect the development of pathogens in the mosquito.

\section{Methods}

\section{Mosquito rearing conditions}

The An. coluzzii used in these experiments were all presumed mated and reared under standard insectary conditions at $27^{\circ} \mathrm{C}, 70-80 \%$ humidity and $12: 12 \mathrm{~h}$ photoperiod with a 1-h dawn and dusk cycle. The VK7 colony was originally collected from Vallee de Kou, Burkina Faso and has been maintained under pyrethroid selection pressure at Liverpool School of Tropical Medicine since 2014 [32, 59]. Resistance in this population is regularly characterised and demonstrates high levels of pyrethroid and DDT resistance [32].

\section{Insecticide exposures}

Pools of 20-30 3-day old adult females from the same generation were exposed for 1 -h to $0.05 \%$ deltamethrin impregnated papers in a WHO tube bioassay as previously described [64]. Mosquitoes used to look at diel cycle and ageing were taken from a different generation to exposed mosquitoes due to high mosquito numbers needed. The starting point for all assays was $10 \mathrm{am}$.

\section{Microarray experiments}

RNA was extracted from pools of 7-10 adult females from unexposed VK7 at the following time points: 3 day old (' 0 h'); 8-h, 12-h, 24-h, 48-h and 72-h and exposed VK7 at the following time points: immediately after $1 \mathrm{~h}$ exposure and then $30 \mathrm{~min}, 1 \mathrm{~h}, 2 \mathrm{~h}, 4 \mathrm{~h}, 8 \mathrm{~h}, 12 \mathrm{~h}, 24 \mathrm{~h}$, $48 \mathrm{~h}$ and $72 \mathrm{~h}$ post exposure. The 0 -h time point was identical for each of the exposed and unexposed groups, and ages are exactly matched e.g. 8-h unexposed corresponds with 8-h post-exposure, and is therefore actually 9-h older than the zero hour control to account for the 1-h exposure. Each biological replicate consisted of pooled RNA, extracted using PicoPure RNA Isolation kit (Arcturus) following manufacturer's instructions. Three or four biological replicates were prepared for each time point for each strain. All biological replicates were taken from the same colony cage; for each time point, all replicates were from mosquitoes that had been exposed to deltamethrin simultaneously. Each timepoint for the exposed mosquitoes was competitively hybridised with the previous time point. The unexposed mosquitoes were competitively hybridised as follows: 0 -h vs 48 -h (ageing); 8 -h vs 12 -h and 12 -h vs 24 -h (two diel time points). The quality of the RNA was assessed using a nanodrop spectrophotometer (Nanodrop Technologies UK) and TapeStation (Agilent). One hundred nanograms of RNA was amplified and labelled with either $C y 3$ and $C y 5$, using the 'Two color low input Quick Amp labelling kit' (Agilent) following manufacturer's instructions. Samples were then purified using the RNA purification kit (Qiagen), with cRNA yield and quality assessed using the spectrophotometer (Nanodrop Technologies UK) and TapeStation (Agilent). Microarray data was obtained from scanning $15 \mathrm{k}$ Agilent Anopheles microarrays (ArrayExpress accession number A-MEXP-2196), hybridised with labelled cRNA, with an Agilent G2205B scanner. Hybridisations were carried out over $17 \mathrm{~h}$ at $65^{\circ} \mathrm{C}$ at $10 \mathrm{rpm}$ rotation and washed following manufacturer's instructions (Agilent). Datasets are available at ArrayExpress: exposure time course (E-MTAB-9422) and ageing time course (E-MTAB-9423). Experimental design is shown in Additional file 11.

\section{Data analysis}

The resulting microarray data was analysed by fitting linear models to normalised corrected signals using the $\mathrm{R}$ package limma [65] following package instructions. Briefly, within and between array normalisation was carried out using loess and Aquantile respectively, with background correction using normexp. Microarrays were analysed both as hybridised using lmFit and eBayes for the unexposed mosquitoes, and also using separate channel analysis for two-colour data allowing comparison to the unexposed control as described in package instructions using intraspotCorrelation, $\operatorname{lmscFit}$ and eBayes (Github: https://github.com/VictoriaIngham/ Time_Course). Benjamini and Hochberg adjusted $p$ value of less than or equal to 0.05 was used for probe significance, no fold change cut-off was applied. Clusters of probes following the same expression patterns were found using Mfuzz [66] with 20 clusters. Mfuzz was selected over k-means clustering as it utilises soft clustering, allowing a membership score to be assigned to each transcript. Optimal fuzzifier value was calculated following published guidelines [67] $(\mathrm{m}=1.30)$, optimal clusters calculated using sum of squares error $(c=7-10) ; c=20$ 
was used to ensure all substructure identified and no between group correlation $>0.9$. All data was standardised so that the expression profile for each transcript had a mean on 0 and a standard deviation of one. All enrichment analyses were carried out using DAVID [68] and KEGG [69] using Benjamini and Hochberg adjusted pvalue of less than or equal to 0.05 for significance. Drosophila homologs were found using VectorBase [70] and FlyBase [71]. Heatmaps were produced using ggplot2, in the case of multiple probes for individual transcripts, these were averaged across all probes. In these cases, significance for multiple probes was defined as at least one of the four probes show significance. Experimental Design visualised in Additional file 11.

\section{Abbreviations}

ITNs: Insecticide Treated Bed Nets; WHO: World Health Organisation; Kdr: Knockdown Resistance; CSPs: Chemosensory Proteins;

P450s: Cytochrome P450s; GSTs: Glutathione-s-transferases; ABCs: ABC transporters; COEs: Carboxylesterase; UGTs: UDP-glucuronyl transferases; TCA: Citrate Cycle; KEGG: Kyoto Encyclopaedia of Genes and Genomes; GO: Gene Ontology; SNP: Single Nucleotide Polymorphism

\section{Supplementary Information}

The online version contains supplementary material available at https://doi. org/10.1186/s12864-021-07646-7.

Additional file 1. Mfuzz clusters for all transcripts. Expression patterns for all transcripts across the 20 soft Mfuzz clusters. Red indicated high cluster membership, blue intermediate and green low. Y-axis indicated normalised expression change and the $x$-axis represents each time point.

Additional file 2. Mfuzz cluster membership and associated enrichments. Cluster ID corresponding to visual display, significant GO term enrichments for each of Biological Processes, Cellular Component and Molecular Function, significant KEGG pathway enrichment and significant InerPro domains. Pattern describes the general pattern seen in the cluster. All $p$-values are shown in brackets with $\mathrm{BH}$ adjustment.

Additional file 3. Transcripts significantly down regulated at all time points. Transcript ID, gene name, gene description, fold change and adjusted $p$-value for each time point.

Additional file 4. Transcripts significantly differential from 1- or 2-h post-exposure onwards. Transcript ID, gene name, gene description, fold change and adjusted $p$-value for each time point. Red indicates nonsignificance.

Additional file 5. Transcripts significantly differential from 4- or 8-h post-exposure onwards. Transcript ID, gene name, gene description, fold change and adjusted $p$-value for each time point. Red indicates nonsignificance.

Additional file 6. Transcripts significantly differential from $24 \mathrm{~h}$ postexposure onwards. Transcript ID, gene name, gene description, fold change and adjusted $p$-value for each time point. Red indicates nonsignificance.

Additional file 7. Changed in pyrethroid resistance-related gene families. Transcript ID, Gene Name and Log2 fold change across all time points for each transcript in the six resistance-related families that are significantly (adjusted $p<=0.05$ ) differential in at least one time point. Black boxes represent non-significant time points. Transcript ID -RX accounts for different splice variants. Heatmap with colour key representing raw fold change for each transcript, in the case of multiple probes the average fold change across all probes. Tabs on the excel sheet represent different families.

Additional file 8. Significant changes in respiratory-related transcripts. Heatmaps showing transcripts involved in (A) Oxidative phosphorylation and (B) TCA cycle that are differential in at least one time point. Pathway membership as defined by KEGG. Transcript ID followed by generic name is shown in row labelling, columns represent different timepoints. Dark grey indicated non-significant.

Additional file 9. Significantly differentially expressed transcripts between 3- and 5-day old females. Transcript ID, Gene Name and Gene Description (VectorBase, April 2020), adjusted $p$-value and fold change for each transcript. Down-regulation indicates transcripts with lower expression in older females, up-regulation is the converse.

Additional file 10. Significantly differentially expressed transcripts between night-time $(11 \mathrm{pm})$ and morning (11 am). Transcript ID, Gene Name and Gene Description (VectorBase, April 2020), adjusted $p$-value and fold change for each transcript. Down-regulation indicates transcripts with higher expression in the evening, up-regulation is the converse.

Additional file 11. Experimental Design. A. Exposure time course, black arrows represent the time at which mosquitoes were harvested for RNA extraction. In the original experimental design, each array was hybridised to the time point before (hyphens). For this paper, limma single channel analysis was used to compare each time point with an unexposed time point (green arrows). WHO tube shows point of exposure. B. Unexposed dataset, dark blue arrows represent direct array hybridisation.

\section{Acknowledgements}

We thank Manuela Bernardi (LSTM) for preparation of Figs. 1 and 4 and John Morgan (LSTM) and Kobie Hyacinthe Toé (Centre National de Recherche et de Formation sur le Paludisme) for original mosquito collections that formed the VK72014 colony.

\section{Authors' contributions}

VAI and HR conceived the study and drafted the manuscript. FB performed all mosquito maintenance, insecticide exposures and RNA extractions. VAI performed the microarray experiments and all subsequent analysis. All authors have read and approved the manuscript.

\section{Funding}

This study, in totality, was funded by an MRC Skills Development fellowship [MR/R024839/1] to VAl.

\section{Availability of data and materials}

The datasets supporting the conclusions of this article are available in the ArrayExpress repository: exposure time course (E-MTAB-9422) and ageing time course (E-MTAB-9423). All R code used in this analysis is available from the corresponding author upon request and at https://github.com/ Victorialngham/Time_Course.

\section{Declarations}

Ethics approval and consent to participate Not Applicable.

\section{Consent for publication}

Not Applicable.

\section{Competing interests}

The authors declare they have no competing interests.

\section{Author details}

'Department of Vector Biology, Liverpool School of Tropical Medicine, Liverpool L35QA, UK. ${ }^{2}$ Present Address: Parasitology Unit, Centre for Infectious Diseases, Universitätsklinikum, Im Neuenheimer Feld 324, 69120 Heidelberg, Germany. ${ }^{3}$ Department of Epidemiology and Population Health, Institute of Infection and Global Health, Faculty of Health and Life Sciences, Leahurst Campus, University of Liverpool, Neston CH647TE, UK. 
Received: 13 August 2020 Accepted: 23 April 2021 Published online: 10 May 2021

\section{References}

1. Bhatt S, Weiss DJ, Cameron E, Bisanzio D, Mappin B, Dalrymple U, et al. The effect of malaria control on Plasmodium falciparum in Africa between 2000 and 2015. Nature. 2015;526(7572):207-11. https://doi. org/10.1038/nature15535.

2. World Malaria Report. 2019. https://www.who.int/publications/i/item/978924 0015791.

3. Staedke SG, Gonahasa S, Dorsey G, Kamya MR, Maiteki-Sebuguzi C, Lynd $A$, et al. Effect of long-lasting insecticidal nets with and without piperonyl butoxide on malaria indicators in Uganda (LLINEUP): a pragmatic, cluster-randomised trial embedded in a national LLIN distribution campaign. Lancet. 2020;395(10232):1292-303. https://doi. org/10.1016/S0140-6736(20)30214-2.

4. Churcher TS, Lissenden N, Griffin JT, Worrall E, Ranson H. The impact of pyrethroid resistance on the efficacy and effectiveness of bednets for malaria control in Africa. Elife. 2016;5:e16090. https://doi.org/10.7554/ eLife.16090.

5. Protopopoff N, Mosha JF, Lukole E, Charlwood JD, Wright A, Mwalimu CD, et al. Effectiveness of a long-lasting piperonyl butoxide-treated insecticidal net and indoor residual spray interventions, separately and together, against malaria transmitted by pyrethroid-resistant mosquitoes: a cluster, randomised controlled, two-by-two fact. Lancet. 2018;391(10130):1577-88. https://doi.org/10.1016/S0140-6736(18)30427-6.

6. Hughes A, Lissenden N, Viana M, Toe KH, Ranson H. Anopheles gambiae populations from Burkina Faso show minimal delayed mortality after exposure to insecticide-treated nets. Parasit Vectors. 2020;13(1):17. https:// doi.org/10.1186/s13071-019-3872-2.

7. Hawley WA, Phillips-Howard PA, ter Kuile FO, Terlouw DJ, Vulule JM, Ombok $M$, et al. Community-wide effects of permethrin-treated bed nets on child mortality and malaria morbidity in western Kenya. Am J Trop Med Hyg. 2003;68(4 Suppl):121-7. https://doi.org/10.4269/ajtmh.2003.68.121.

8. Killeen GF, Smith TA. Exploring the contributions of bed nets, cattle, insecticides and excitorepellency to malaria control: a deterministic model of mosquito host-seeking behaviour and mortality. Trans R Soc Trop Med Hyg. 2007;101(9):867-80. https://doi.org/10.1016/j.trstmh.2007.04.022.

9. Killeen GF, Okumu FO, N'Guessan R, Coosemans M, Adeogun A, Awolola S, et al. The importance of considering community-level effects when selecting insecticidal malaria vector products. Parasit Vectors. 2011;4(1):160. https://doi.org/10.1186/1756-3305-4-160.

10. Tiono AB, Ouédraogo A, Ouattara D, Bougouma EC, Coulibaly S, Diarra A, et al. Efficacy of Olyset duo, a bednet containing pyriproxyfen and permethrin, versus a permethrin-only net against clinical malaria in an area with highly pyrethroid-resistant vectors in rural Burkina Faso: a clusterrandomised controlled trial. Lancet. 2018;392(10147):569-80. https://doi. org/10.1016/S0140-6736(18)31711-2.

11. Martinez-Torres D, Chandre F, Williamson MS, Darriet F, Berge JB, Devonshire $\mathrm{AL}$, et al. Molecular characterization of pyrethroid knockdown resistance $(\mathrm{kdr})$ in the major malaria vector Anopheles gambiae ss. Insect Mol Biol. 1998;7(2):179-84. https://doi.org/10.1046/j.1365-2583.1998.72062.x.

12. Balabanidou V, Kampouraki A, MacLean M, Blomquist GJ, Tittiger C, Juárez MP, et al. Cytochrome P450 associated with insecticide resistance catalyzes cuticular hydrocarbon production in Anopheles gambiae. Proc Natl Acad Sci. 2016;113(33):9268-73. https://doi.org/10.1073/pnas.1608295113.

13. Ingham VAVA, Anthousi A, Douris V, Harding NJNJ, Lycett G, Morris M, et al. A sensory appendage protein protects malaria vectors from pyrethroids. Nature. 2019;577(7790):376-80. https://doi.org/10.1038/s41586-019-1864-1.

14. Müller P, Warr E, Stevenson BJ, Pignatelli PM, Morgan JC, Steven A, et al. Field-caught permethrin-resistant anopheles gambiae overexpress CYP6P3, a P450 that metabolises pyrethroids. PLoS Genet. 2008:4(11):e1000286. https://doi.org/10.1371/journal.pgen.1000286.

15. Stevenson BJ, Bibby J, Pignatelli P, Muangnoicharoen S, O'Neill PM, Lian L-Y, et al. Cytochrome P450 6M2 from the malaria vector Anopheles gambiae metabolizes pyrethroids: sequential metabolism of deltamethrin revealed. Insect Biochem Mol Biol. 2011:41(7):492-502. https://doi.org/10.1016/j. ibmb.2011.02.003.

16. Yunta C, Hemmings K, Stevenson B, Koekemoer LL, Matambo T, Pignatelli $P$, et al. Cross-resistance profiles of malaria mosquito P450s associated with pyrethroid resistance against WHO insecticides. Pestic Biochem Physiol. 2019;161:61-7. https://doi.org/10.1016/j.pestbp.2019.06.007.

17. Riveron JM, Yunta C, Ibrahim SS, Djouaka R, Irving H, Menze BD, et al. A single mutation in the GSTe2 gene allows tracking of metabolically based insecticide resistance in a major malaria vector. Genome Biol. 2014;15(2):R27. https://doi.org/10.1186/gb-2014-15-2-r27.

18. Pignatelli $P$, Ingham VAA, Balabanidou V, Vontas J, Lycett $G$, Ranson $H$. The Anopheles gambiae ATP-binding cassette transporter family: phylogenetic analysis and tissue localization provide clues on function and role in insecticide resistance. Insect Mol Biol. 2018;27(1):110-22. https://doi.org/1 $0.1111 /$ imb.12351.

19. Feng $X$, Li M, Liu N. Carboxylesterase genes in pyrethroid resistant house flies, Musca domestica. Insect Biochem Mol Biol. 2018;92:30-9. https://doi. org/10.1016/j.ibmb.2017.11.007.

20. Ahn S-J, Vogel H, Heckel DG. Comparative analysis of the UDPglycosyltransferase multigene family in insects. Insect Biochem Mol Biol. 2012;42(2):133-47. https://doi.org/10.1016/j.ibmb.2011.11.006.

21. Ingham VA, Wagstaff $S$, Ranson $H$. Transcriptomic meta-signatures identified in Anopheles gambiae populations reveal previously undetected insecticide resistance mechanisms. Nat Commun. 2018;9(1):5282. https://doi.org/10.103 8/s41467-018-07615-x.

22. Thiévent K, Hauser G, Elaian O, Koella JC. The interaction between permethrin exposure and malaria infection affects the host-seeking behaviour of mosquitoes. Malar J. 2019;18(1):79. https://doi.org/10.1186/s12 936-019-2718-x.

23. Gong Y, Li T, Zhang L, Gao X, Liu N. Permethrin induction of multiple cytochrome $P 450$ genes in insecticide resistant mosquitoes, Culex quinquefasciatus. Int J Biol Sci. 2013;9(9):863-71. https://doi.org/10.7150/ijbs. 6744

24. Liu N, Li T, Reid WR, Yang T, Zhang L. Multiple cytochrome P450 genes: their constitutive overexpression and permethrin induction in insecticide resistant mosquitoes, Culex quinquefasciatus. PLoS One. 2011;6(8):e23403. https://doi.org/10.1371/journal.pone.0023403.

25. Festucci-Buselli RA, Carvalho-Dias AS, de Oliveira-Andrade M, Caixeta-Nunes C, Li H-M, Stuart JJ, et al. Expression of Cyp6g1 and Cyp12d1 in DDT resistant and susceptible strains of Drosophila melanogaster. Insect Mol Biol. 2005;14(1):69-77. https://doi.org/10.1111/j.1365-2583.2005.00532.x.

26. Mastrantonio V, Ferrari M, Negri A, Sturmo T, Favia G, Porretta D, et al. Insecticide exposure triggers a modulated expression of $A B C$ transporter genes in larvae of Anopheles gambiae s.s. Insects. 2019;10:66.

27. Hu B, Zhang S-H, Ren M-M, Tian X-R, Wei Q, Mburu DK, et al. The expression of Spodoptera exigua P450 and UGT genes: tissue specificity and response to insecticides. Insect Sci. 2019;26(2):199-216. https://doi.org/10.1111/17447917.12538

28. Hu F, Dou W, Wang J-J, Jia F-X, Wang J-J. Multiple glutathione S-transferase genes: identification and expression in oriental fruit fly, Bactrocera dorsalis. Pest Manag Sci. 2014;70(2):295-303. https://doi.org/10.1002/ps.3558.

29. Ingham VA, Pignatelli $P$, Moore JD, Wagstaff $S$, Ranson $H$. The transcription factor Maf-S regulates metabolic resistance to insecticides in the malaria vector Anopheles gambiae. BMC Genomics. 2017;18(1):669. https://doi.org/1 0.1186/s12864-017-4086-7.

30. Misra JR, Lam G, Thummel CS. Constitutive activation of the Nrf2/Keap1 pathway in insecticide-resistant strains of Drosophila. Insect Biochem Mol Biol. 2013;43:1116-24. https://doi.org/10.1016/j.ibmb.2013.09.005.

31. Misra JR, Horner MA, Lam G, Thummel CS. Transcriptional regulation of xenobiotic detoxification in Drosophila. Genes Dev. 2011;25(17):1796-806. https://doi.org/10.1101/gad.17280911.

32. Williams J, Flood L, Praulins G, Ingham VA, Morgan J, Lees RS, et al. Characterisation of Anopheles strains used for laboratory screening of new vector control products. Parasit Vectors. 2019;12(1):522. https://doi.org/10.11 86/s13071-019-3774-3.

33. Wang X, Martínez M-A, Dai M, Chen D, Ares I, Romero A, et al. Permethrininduced oxidative stress and toxicity and metabolism. A review. Environ Res. 2016;149:86-104. https://doi.org/10.1016/j.envres.2016.05.003.

34. Ingaramo MC, Sánchez JA, Dekanty A. Regulation and function of p53: a perspective from Drosophila studies. Mech Dev. 2018;154:82-90. https://doi. org/10.1016/j.mod.2018.05.007.

35. Nakamura M, Baldwin D, Hannaford S, Palka J, Montell C. Defective proboscis extension response (DPR), a member of the Ig superfamily required for the gustatory response to salt. J Neurosci. 2002;22:3463 LP-472. https://doi.org/10.1523/JNEUROSCI.22-09-03463.2002. 
36. Jordan KW, Craver KL, Magwire MM, Cubilla CE, Mackay TFC, Anholt RRH. Genome-wide association for sensitivity to chronic oxidative stress in Drosophila melanogaster. PLoS One. 2012;7(6):e38722. https://doi.org/10.13 71/journal.pone.0038722

37. Yunta C, Grisales N, Nász S, Hemmings K, Pignatelli P, Voice M, et al. Pyriproxyfen is metabolized by P450s associated with pyrethroid resistance in an. gambiae. Insect Biochem Mol Biol. 2016;78:50-7. https://doi.org/10.1 016/j.ibmb.2016.09.001.

38. Vontas J, Grigoraki L, Morgan J, Tsakireli D, Fuseini G, Segura L, et al. Rapid selection of a pyrethroid metabolic enzyme CYP9K1 by operational malaria control activities. Proc Natl Acad Sci. 2018;115:4619 LP-4624 http://www. pnas.org/content/115/18/4619.abstract.

39. Chiu T-L, Wen Z, Rupasinghe SG, Schuler MA. Comparative molecular modeling of Anopheles gambiae CYP6Z1, a mosquito P450 capable of metabolizing DDT. Proc Natl Acad Sci. 2008;105(26):8855-60. https://doi. org/10.1073/pnas.0709249105.

40. Huang Y, Guo Q, Sun X, Zhang C, Xu N, Xu Y, et al. Culex pipiens pallens cuticular protein CPLCG5 participates in pyrethroid resistance by forming a rigid matrix. Parasit Vectors. 2018;11(1):6. https://doi.org/10.1186/s13071-01 7-2567-9.

41. Jones CM, Sanou A, Guelbeogo WM, Sagnon N, Johnson PCD, Ranson H. Aging partially restores the efficacy of malaria vector control in insecticideresistant populations of Anopheles gambiae s.l. from Burkina Faso. Malar J. 2012;11:24. https://doi.org/10.1186/1475-2875-11-24.

42. Chouaibou MS, Chabi J, Bingham GV, Knox TB, N'Dri L, Kesse NB, et al. Increase in susceptibility to insecticides with aging of wild Anopheles gambiaemosquitoes from Côte d'Ivoire. BMC Infect Dis. 2012;12(1):214. https://doi.org/10.1186/1471-2334-12-214.

43. Balmert NJ, Rund SSC, Ghazi JP, Zhou P, Duffield GE. Time-of-day specific changes in metabolic detoxification and insecticide resistance in the malaria mosquito Anopheles gambiae. J Insect Physiol. 2014;64:30-9. https://doi. org/10.1016/j.jinsphys.2014.02.013.

44. Rund SSC, Hou TY, Ward SM, Collins FH, Duffield GE. Genome-wide profiling of diel and circadian gene expression in the malaria vector Anopheles gambiae. Proc Natl Acad Sci. 2011;108:E421 LP-E430. https://doi.org/10.1 073/pnas.1100584108.

45. Malla Reddy P, Bashamohideen M. Modulations in the levels of respiration and ions in carp Cyprinus carpio (L.) exposed to cypermethrin. Environ Monit Assess. 1995;35:221-6. https://doi.org/10.1007/BF00547633.

46. Gassner B, Wüthrich A, Scholtysik G, Solioz M. The pyrethroids permethrin and cyhalothrin are potent inhibitors of the mitochondrial complex I. J Pharmacol Exp Ther. 1997;281:855 LP-860 http://jpet.aspetjournals.org/ content/281/2/855.abstract.

47. Braguini WL, Cadena SMSC, Carnieri EGS, Rocha MEM, de Oliveira MBM. Effects of deltamethrin on functions of rat liver mitochondria and on native and synthetic model membranes. Toxicol Lett. 2004;152(3):191-202. https:// doi.org/10.1016/j.toxlet.2004.03.017.

48. Oliver SV, Brooke BD. The role of oxidative stress in the longevity and insecticide resistance phenotype of the major malaria vectors Anopheles arabiensis and Anopheles funestus. PLoS One. 2016;11(3):e0151049. https:// doi.org/10.1371/journal.pone.0151049.

49. Fetoui H, Feki A, Ben SG, Kamoun H, Fakhfakh F, Gdoura R. Exposure to lambda-cyhalothrin, a synthetic pyrethroid, increases reactive oxygen species production and induces genotoxicity in rat peripheral blood. Toxicol Ind Health. 2013;31(5):433-41. https://doi.org/10.1177/0748233713475516.

50. Parker JEA, Angarita-Jaimes N, Abe M, Towers CE, Towers D, McCall PJ. Infrared video tracking of Anopheles gambiae at insecticide-treated bed nets reveals rapid decisive impact after brief localised net contact. Sci Rep. 2015;5(1):13392. https://doi.org/10.1038/srep13392.

51. Murray GPD, Lissenden N, Jones J, Voloshin V, Toé KH, Sherrard-Smith E, et al. Barrier bednets target malaria vectors and expand the range of usable insecticides. Nat Microbiol. 2019;5(1):40-7. https://doi.org/10.1038/s41564-01 9-0607-2.

52. Oliver SV, Brooke BD. The effect of multiple blood-feeding on the longevity and insecticide resistant phenotype in the major malaria vector Anopheles arabiensis (Diptera: Culicidae). Parasit Vectors. 2014;7(1):390. https://doi.org/1 0.1186/1756-3305-7-390.

53. Faust JE, Verma A, Peng C, McNew JA. An inventory of peroxisomal proteins and pathways in Drosophila melanogaster. Traffic. 2012;13(10):1378-92. https://doi.org/10.1111/j.1600-0854.2012.01393.x.
54. Chen T-H, Wu Y-J, Hou J-N, Chiu C-H, Chen W-J. The p53 gene with emphasis on its paralogues in mosquitoes. J Microbiol Immunol Infect. 2017;50(6):747-54. https://doi.org/10.1016/j.jmii.2017.06.006.

55. Zhou Y, Fu W-B, Si F-L, Yan Z-T, Zhang Y-J, He Q-Y, et al. UDPglycosyltransferase genes and their association and mutations associated with pyrethroid resistance in Anopheles sinensis (Diptera: Culicidae). Malar J. 2019;18(1):62. https://doi.org/10.1186/s12936-019-2705-2.

56. O'Keefe LV, Colella A, Dayan S, Chen Q, Choo A, Jacob R, et al. Drosophila orthologue of WWOX, the chromosomal fragile site FRA16D tumour suppressor gene, functions in aerobic metabolism and regulates reactive oxygen species. Hum Mol Genet. 2010;20(3):497-509. https://doi.org/10.1 093/hmg/ddq495

57. Isaacs AT, Mawejje HD, Tomlinson S, Rigden DJ, Donnelly MJ. Genome-wide transcriptional analyses in Anopheles mosquitoes reveal an unexpected association between salivary gland gene expression and insecticide resistance. BMC Genomics. 2018;19(1):225. https://doi.org/10.1186/s12864-01 8-4605-1.

58. Elanga-Ndille E, Nouage L, Binyang A, Assatse T, Tene-Fossog B, Tchouakui $M$, et al. Overexpression of two members of D7 salivary genes family is associated with pyrethroid resistance in the malaria vector Anopheles funestus ss but not in Anopheles gambiae in Cameroon. Genes (Basel). 2019; 10:211.

59. Namountougou M, Simard F, Baldet T, Diabaté A, Ouédraogo JB, Martin T, et al. Multiple insecticide resistance in Anopheles gambiae sl populations from Burkina Faso, West Africa. PLoS One. 2012;7(11):e48412. https://doi. org/10.1371/journal.pone.0048412.

60. Akbari OS, Antoshechkin I, Amrhein H, Williams B, Diloreto R, Sandler J, et al. The developmental transcriptome of the mosquito Aedes aegypti, an invasive species and major arbovirus vector. G3 Genes|Genomes|Genetics. 2013;3:1493-509. https://doi.org/10.1534/g3.113.006742.

61. Marinotti O, Calvo E, Nguyen QK, Dissanayake S, Ribeiro JMC, James AA. Genome-wide analysis of gene expression in adult Anopheles gambiae. Insect Mol Biol. 2006;15(1):1-12. https://doi.org/10.1111/j.1365-2583.2006. 00610.x

62. Molina-Cruz A, DeJong RJ, Charles B, Gupta L, Kumar S, Jaramillo-Gutierrez $\mathrm{G}$, et al. Reactive oxygen species modulate Anopheles gambiae immunity against bacteria and Plasmodium. J Biol Chem. 2008;283(6):3217-23. https:// doi.org/10.1074/jbc.M705873200.

63. Castillo JC, Ferreira ABB, Trisnadi N, Barillas-Mury C. Activation of mosquito complement antiplasmodial response requires cellular immunity. Sci Immunol. 2017;2:eaal1505. https://doi.org/10.1126/sciimmunol.aal1505.

64. Organization $\mathrm{WH}$. Test procedures for insecticide resistance monitoring in malaria vector mosquitoes. 2016.

65. Ritchie ME, Phipson B, Wu D, Hu Y, Law CW, Shi W, et al. limma powers differential expression analyses for RNA-sequencing and microarray studies. Nucleic Acids Res. 2015;43(7):e47. https://doi.org/10.1093/nar/gkv007.

66. Kumar L, Futschik ME. Mfuzz: a software package for soft clustering of microarray data. Bioinformation. 2007;2(1):5-7. https://doi.org/10.6026/9732 0630002005

67. Schwammle $V$, Jensen ON. A simple and fast method to determine the parameters for fuzzy c-means cluster analysis. Bioinformatics. 2010;26(22): 2841-8. https://doi.org/10.1093/bioinformatics/btq534.

68. Huang DW, Sherman BT, Lempicki RA. Systematic and integrative analysis of large gene lists using DAVID bioinformatics resources. Nat Protoc. 2008:4(1): 44-57. https://doi.org/10.1038/nprot.2008.211.

69. Kanehisa M, Goto S. KEGG: Kyoto encyclopedia of genes and genomes. Nucleic Acids Res. 2000;28(1):27-30. https://doi.org/10.1093/nar/28.1.27.

70. Giraldo-Calderón Gl, Emrich SJ, MacCallum RM, Maslen G, Dialynas E, Topalis $P$, et al. VectorBase: an updated bioinformatics resource for invertebrate vectors and other organisms related with human diseases. Nucleic Acids Res. 2014:43:D707-13.

71. Consortium F. The FlyBase database of the Drosophila genome projects and community literature. Nucleic Acids Res. 2003;31:172-5.

\section{Publisher's Note}

Springer Nature remains neutral with regard to jurisdictional claims in published maps and institutional affiliations. 